\title{
Cell on Wheels-Unmanned Aerial Vehicle System for Providing Wireless Coverage in Emergency Situations
}

\author{
Hazim Shakhatreh $\mathbb{D},{ }^{1}$ Khaled Hayajneh $\mathbb{D}^{\mathbb{D}},{ }^{1}$ Khaled Bani-Hani $\mathbb{D}^{1},{ }^{1}$ Ahmad Sawalmeh $\mathbb{D},{ }^{2,3}$ \\ and Muhammad Anan (iD) \\ ${ }^{1}$ Department of Telecommunications Engineering, Hijjawi Faculty for Engineering Technology, \\ Yarmouk University, Irbid, Jordan \\ ${ }^{2}$ Computer Science Department, Northern Border University, Arar, Saudi Arabia \\ ${ }^{3}$ Remote Sensing Unit, Northern Border University, Arar 91431, Saudi Arabia \\ ${ }^{4}$ Software Engineering Department, Alfaisal University, Riyadh 11533, Saudi Arabia \\ Correspondence should be addressed to Hazim Shakhatreh; hazim.s@yu.edu.jo
}

Received 5 October 2021; Revised 1 November 2021; Accepted 6 November 2021; Published 22 November 2021

Academic Editor: Chao Liu

Copyright (c) 2021 Hazim Shakhatreh et al. This is an open access article distributed under the Creative Commons Attribution License, which permits unrestricted use, distribution, and reproduction in any medium, provided the original work is properly cited.

\begin{abstract}
Due to natural disasters, unmanned aerial vehicles (UAVs) can be deployed as aerial wireless base stations when conventional cellular networks are out of service. They can also supplement the mobile ground station to provide wireless devices with improved coverage and faster data rates. Cells on wheels (CoWs) can also be utilized to provide enhanced wireless coverage for short-term demands. In this paper, a single CoW cooperates with a single UAV in order to provide maximum wireless coverage to ground users. The optimization problem is formulated to find the following: (1) the optimal 2D placement of the CoW, (2) the optimal 3D placement of the UAV, (3) the optimal bandwidth allocation, (4) the percentage of the available bandwidth that must be provided to the CoW and UAV, and (5) the priority of wireless coverage; which maximizes the number of covered users. We utilize the exhaustive search (ES) and particle swarm optimization (PSO) algorithms to solve the optimization problem. The effectiveness of the proposed algorithms is validated using simulation results.
\end{abstract}

\section{Introduction}

UAV networks have been developed as a possible technology of quickly providing wireless coverage to a geographic area, where a flying UAV can be quickly deployed to operate as a cell site $[1,2]$. The advantage of deploying UAVs as flying base stations over traditional terrestrial base stations is their ability to change altitude, avoid obstacles, and increase the possibility of establishing line of sight (LoS) communication connections with wireless devices $[3,4]$. UAVs are especially useful in situations when the conventional cellular network is either unavailable or requires assistance to offer the necessary capacity and coverage $[5,6]$. Verizon, for example, has developed an airborne long-term evolution (LTE) service to provide 4G-LTE connectivity during disaster recovery exercises and emergency management [7]. The authors in [8] study the emergency UAV deployment to minimize the UAV deployment delay until covering the entire geographical area by considering the UAVs' different flying speeds and altitudes during the deployment. In [9], the authors establish a unified framework for a UAV-assisted emergency network. The scheduling and trajectory of drones are jointly optimized to provide wireless connectivity to wireless devices with surviving ground mobile stations. The authors in [10] propose a deployment tool for a drone-aided emergency network to provide wireless device coverage in a large-scale disaster scenario. LTE femtocell base stations are mounted on drones and deployed to their assigned placement. Such a network's deployment tool takes into account the characteristics of the UAVs (such as battery life and flight time), user requirements, and a $3 \mathrm{D}$ representation of the environment. In [11], the authors propose energy-efficient planning of drones for emergencies. Their problem formulation considers both outdoor and indoor users, where 
the objective is to find the placements of multiple drones that provide wireless coverage based on the locations and distribution of users. In addition, supplementing the surviving communication infrastructure and keeping first responders connected to their command centers, a portable cell or CoW can be utilized [12]. One can establish a temporary network to enable important public safety communication throughout the disaster area by efficiently deploying these mobile cells. These portable cells, which are simple to deploy, configure, and adapt, are an ideal solution for any disaster response effort. The authors in [12] present a decentralized relocation algorithm that enables CoWs to change their locations in response to the potential mobility of ground users. In [13], the authors describe and propose a portable mobile wireless network infrastructure that is ideally suited for emergency response and disaster recovery operations. The authors in [14] study the challenges of emergency communication networks for disaster response. These challenges include popularity, usability, capacity, operability, reliability, and adaptability. In [15], the authors demonstrate that drone assistance in CoW-UAV wireless networks can provide significant advantages to mmWave backhaul under certain system parameters. The authors in [16] proposed an efficient 3D placement of a single UAV for assisting terrestrial wireless networks. In their proposed model, a single UAV is utilized to assist the GBS and provide wireless coverage for arbitrarily distributed ground terminals, considering the impact of the obstacle blockage for the A2G path loss model. The authors in [17] propose a framework that combines UAV support with wirelesspowered communication techniques to further improve energy efficiency in distributed nonorthogonal multiple access public safety networks. In [18], the authors propose a distributed user-centric risk-aware resource management framework in a UAV-assisted public safety network supported by both a static UAV and a mobile UAV. The authors in [19] introduce a resource orchestration framework in a UAV-assisted wireless-powered communication network within a public safety system, based on the principles of contract theory and reinforcement learning.

In this paper, a single $\mathrm{CoW}$ and a single UAV are utilized to maximize the wireless communication coverage in emergency situations. The objective of the proposed optimization problem is to find the optimal 2D placement of the CoW, the optimal 3D placement of the UAV, optimal bandwidth allocation to the UAV and CoW, and the priority of wireless coverage that maximizes the number of covered wireless devices. To the best of our knowledge, this is the first work that proposes using a CoW-UAV system to maximize the wireless coverage in emergency situations. The main contributions of this paper are summarized as follows:

(1 )Realistic path loss models for a CoW-UAV system are presented, and the trade-off introduced by these models is described

(2) The optimization problem for a CoW-UAV system is formulated with the objective of maximizing the wireless coverage
(3) Exhaustive search (ES) and particle swarm optimization (PSO) algorithms are utilized to solve the optimization problem

The remainder of this paper is organized as follows. In Section 2, the system model is described. Then, in Section 3, the optimization problem is formulated, where the goal is to maximize the number of covered wireless devices. Next, the proposed algorithms are presented to solve the optimization problem in Section 4. The numerical results are presented in Section 5. Finally, Section 6 concludes the paper.

\section{System Model}

Consider an urban area denoted as $\mathbb{E} \in(A \times A)$ served by a CoW assisted by a UAV that acts as an aerial base station, as shown in Figure 1. Both the CoW and UAV have fixed transmission power. Let $\left(x_{c}, y_{c}, 6 \mathrm{~m}\right)$ denote the $2 \mathrm{D}$ placement of the CoW, and let $\left(x_{u}, y_{u}, z_{u}\right)$ denote the 3D placement of the UAV, as shown in Figure 1. The movement of the UAV is restricted to specific heights according to the law restrictions, while the roads on the given area restrict the movement of the CoW. It is assumed that all users are located inside the urban environment, and use $\left(x_{i}, y_{i}, 0\right)$ to denote the placement of user $i \in I$. The CoW or the UAV serves the user's wireless device $i$ if the quality of service (QoS), measured by the signal-to-noise ratio (SNR), is above a predefined threshold. Such a threshold depends on the type of service (e.g., voice, data, and video) required by the user.

The CoW and the UAV are equipped with transceiver antennas, and a downlink scenario is envisioned, in which the frequency division multiple access (FDMA) technology is used to transfer data and provide coverage for wireless devices. As a result, each wireless device has its own dedicated communication channel, and there is no interference or channel overlap between CoW and UAV channels. The bandwidths of the CoW and the UAV are $B_{1}$ and $B_{2}$, respectively. The CoW and the UAV are supported by backhaul links that connect them to Internet's core. The highest benefit from CoW and UAV deployment may be obtained by finding the optimal placements for them. Assuming that all wireless devices have the same QoS, the highest benefit can be realized by connecting the CoW and the UAV to as many wireless devices as possible. The CoW and the UAV placements affect both the number of served wireless devices and the quality of the channel between each wireless device and the base station (CoW or UAV). The critical question in this context that we need to answer is which base station starts the wireless coverage first, CoW or UAV? In the downlink scenario, we consider two types of channels: CoW to wireless device channel and UAV to wireless device channel.

2.1. CoW to Wireless Device Channel. In this paper, we utilize the independent Rayleigh fading model to represent the time-varying channel between the CoW and a wireless device $i$ in an urban environment [20,21]. Time variation occurs when the status of obstacles between the transmitter and the receiver is uncertain due to the movement of the obstacles. Changes in the amplitudes, delays, and quantity of 


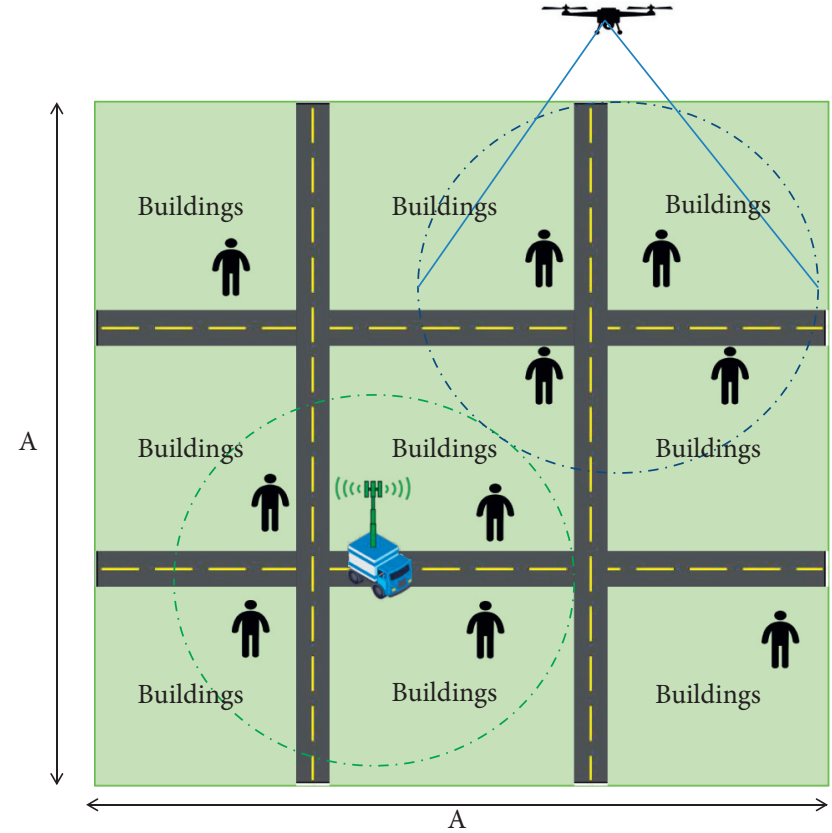

Figure 1: The proposed system model. The area $(\mathrm{Ax} \mathrm{A})$ is covered by a CoW assisted by a UAV.

multipath components related to each signal can thus be detected. Over a much longer time scale, these changes result in a constructive and destructive addition of multipath components. The received power of wireless device $i$ served by the $\mathrm{CoW}$ is formulated as follows:

$$
P_{r_{i, 1}}[\mathrm{~d} B m]=P_{C o W}(\mathrm{~d} B m)+20 \log \left(g_{i}\right)-10 n \log \left(d_{(i, 1)}\right)
$$

where $P_{C o W}$ is the transmitted power by CoW in $\mathrm{dBm}, g_{i}$ is the Rayleigh fading power for the wireless device $i$ in $\mathrm{dB}$, and $d_{(i, 1)}$ is the distance between the CoW and the wireless device $i$ which is given by

$$
d_{(i, 1)}=\sqrt{\left(x_{i}-x_{c}\right)^{2}+\left(y_{i}-y_{c}\right)^{2}} .
$$

2.2. UAV to Wireless Device Channel. For the downlink communication between the UAV and wireless devices, we use the air-to-ground channel model, which is one of the most commonly used radio propagation models [22]. In general, it is dependent on the line of sight (LoS) and nonline of sight (NLoS) links, as well as their respective chance of occurrence. This channel model takes into account two types of propagation: LoS propagation and NLoS propagation. Based on the environment and the placement of the UAV, the following formula is used to calculate the probability of getting a LoS signal:

$$
P_{\text {LoS }}=\frac{1}{1+\alpha \exp [-\beta(\theta-\alpha)]},
$$

where $\alpha$ and $\beta$ are constants, and their values are affected by the environment, $\theta=\arctan \left(h_{u} / r_{(u, i)}\right)$ is the elevation angle from the UAV to wireless device $i, h_{u}$ is the UAV altitude, and $r_{(u, i)}$ is the horizontal distance between the projection of the UAV placement on the ground and the placement of wireless device $i$. The trade-off in this model was described by the authors of [23]. The path loss between the UAV and the wireless device decreases as the altitude decreases, while the probability of line of sight links also decreases. On the other hand, line of sight links occur with a high probability at high altitudes, while path loss increases. Taking into account the LoS and NLoS link, the total average path loss in this model can be found as follows:

$$
P L\left(h_{u}, r_{u, i}\right)(\mathrm{d} B)=10 n \log \left(\frac{4 \pi f_{c} d_{i, 2}}{c}\right)+P_{\mathrm{Los}} \xi_{\mathrm{LoS}}+P_{\mathrm{NLos}} \xi_{\mathrm{NLoS}}
$$

where the first term represents the free-space path loss, $f_{c}$ is the carrier frequency, $c$ is the speed of light, $n$ is the path loss exponent, $\mathrm{d}_{(i, 2)}$ is the distance between the UAV and wireless device $i$, and $\xi_{\mathrm{LoS}}$ and $\xi_{\mathrm{NLOS}}$ are the average additional losses for LoS and NLoS links in $\mathrm{dB}$, respectively. These losses are environment-dependent.

The received power of wireless device $i$ served by the $\mathrm{UAV}$ is given by

$$
r_{(i, 2)}(\mathrm{d} B m)=P_{u}(\mathrm{~d} B m)-P L\left(h_{u}, r u, i\right)(\mathrm{d} B),
$$

where $P_{u}$ is the transmitted power by UAV in $\mathrm{dBm}$ and $P L\left(h_{u}, r_{(u, i)}\right)$ is the total average path loss in $\mathrm{dB}$.

\section{Problem Formulations}

The received $\operatorname{SNR}\left(\operatorname{SNR}_{(i, q)}\right)$ of wireless device $i \in I$ from the $\operatorname{CoW}(q=1)$ or the UAV $(q=2)$ is given by

$$
\operatorname{SNR}_{(i, q)}=\frac{\operatorname{Pr}_{(i, q)}}{N_{i}}, \quad i \in I, q \in\{1,2\},
$$

where $\operatorname{Pr}_{(i, q)}$ is the received power at a wireless device $i \in I$ from the q-th base station and $N_{i}$ is the channel noise power, which is given by $N_{i}=N_{o} b_{(i, q)}$, in which $N_{o}$ is the power spectral density of noise channel and $b_{(i, q)}$ is the bandwidth allocated for a wireless device $i \in I$ from base station $q \in\{1,2\}$.

The main goal of this paper is to find the optimal placements for the CoW and the UAV that maximize the number of served wireless devices, as illustrated in Figure 1. To formulate the optimization problem, we define the binary variable $V_{(i, q)}, i \in I$ and $q \in 1,2$ which ensures whether a wireless device $i \in I$ is served by base station $q \in\{1,2\}$ or not. The binary variable $V_{(i, q)}$ takes value 1 if $\mathrm{SNR}_{(i, q)} \geq \mathrm{SNR}_{t h}$, and it takes value 0 otherwise, where $\mathrm{SNR}_{\text {th }}$ is the threshold SNR.

Then, if wireless device $i \in I$ is served, $\mathrm{SNR}_{(i, q)} \geq \mathrm{SNR}_{t h}$ must be satisfied. This constraint can be further formulated as follows:

$$
\mathrm{SNR}_{(i, q)} \geq \mathrm{SNR}_{\mathrm{th}}-A\left(1-V_{(i, q)}\right),
$$

where $A$ is significantly constant, greater than the maximum value of $\mathrm{SNR}_{\mathrm{th}}$. The bandwidth allocated to each base station 
(either CoW or UAV) cannot exceed its available bandwidth. Therefore,

$$
\sum_{i=1}^{|I|} b_{(i, q)} V_{(i, q)} \leq B_{q}, \quad \forall q \in\{1,2\},
$$

where $b_{(i, q)}$ is the bandwidth allocated to a wireless device $i \in I$ from base station $q \in 1,2$. Here, each base station's bandwidth is not fixed; therefore, it must be optimized. Many factors influence the optimal bandwidth, including available bandwidth, QoS requirement, wireless device demands, environment, and scenario.

The bandwidth allocated for each BS should be obtained in this research work in order to serve as many wireless devices from the total number of devices in the urban area as possible. Hence, we define the variable $\gamma, \gamma \in[0,1]$, which indicates how much bandwidth should be allocated to CoW. The bandwidth allotted to $\mathrm{CoW}$ is calculated as follows: $B_{1}=\gamma B$, while the bandwidth allotted to UAV is specified as follows: $B_{2}=(1-\gamma) B$. The optimization problem aims to find the following: (1) the optimal 2D placement of the CoW, (2) the optimal 3D placement of the UAV, (3) optimal bandwidth allocation, and (4) the priority of wireless coverage, which maximize the number of covered users. The optimization problem is given by

$$
\underset{\left(\left(x_{c}, y_{c}\right),\left(x_{u}, y_{u}, z_{u}\right), \gamma, V_{(i, q)}\right)}{\operatorname{maximize}} \sum_{i=1}^{|I|} \sum_{q=1}^{2} V_{(i, q)},
$$

Subject to $\mathrm{SNR}_{i, q} \geq \mathrm{SNR}_{\mathrm{th}}-A\left(1-V_{(i, q)}\right), \forall i \in I, q \in\{1,2\}$,

$$
\begin{aligned}
& \sum_{i=1}^{|I|} b_{(i, q)} V_{(i, q)} \leq B_{q}, \quad \forall q \in\{1,2\} \\
& \sum_{q=1}^{2} V_{(i, q)} \leq 1, \quad \forall i \in I \\
& B_{1}=\gamma B \\
& B_{2}=(1-\gamma) B \\
& 0 \leq \gamma \leq 1 \\
& \left(x_{c}, y_{c}\right) \in R_{c}^{2} \\
& \left(x_{c}, y_{c}, z_{u}\right) \in R_{u}^{3}
\end{aligned}
$$

The first constraint set ensures that the QoS of each wireless device should be greater or equal to $\mathrm{SNR}_{\text {th }}$ to be served from either the CoW or the UAV. The second constraint set guarantees that the sum of wireless devices bandwidths does not exceed the base station bandwidth. The third constraint set ensures that a wireless device should be connected to one base station at most or none of them. The constraint sets $9 \mathrm{e}-9 \mathrm{f}-9 \mathrm{~g}$ represent the bandwidth that should be allocated to each base station. The constraint sets 9h-9i represent the allowable values for $2 \mathrm{D}$ and $3 \mathrm{D}$ placements of the CoW and the UAV, respectively.

\section{Methodology}

This section presents methods of finding the solution of CoW and UAV positions and the percentage of the allocated bandwidth for each one. This work employs exhaustive search and PSO algorithms to find the maximum number of served users in the proposed area.

4.1. Exhaustive Search (ES). The exhaustive search is considered as optimal solution. It can find the best $2 \mathrm{D}$ position of CoW, 3D position of UAV, and the available bandwidth at each one of them. Considering the priority, the BS (i.e., CoW or UAV) that has the priority is assumed to be $B S_{1}$, and the other one is $B S_{2}$. Firstly, the $B S_{1}$ will be deployed in allowable positions and serve a set of users (U) from I. Secondly, the $B S_{2}$ will be deployed to serve as much users as possible from set $(|I|-|U|)$. Determining the placements of $B S_{1}, B S_{2}$ will be synchronized at each search point. The ES algorithm will require a lot of calculations, and possibly even impossible in practice. The PSO algorithm is employed to overcome the high computational overhead issue in ES.

4.2. PSO. The PSO algorithm is being used in the study to provide a solution $[24,25]$. The PSO is considered a suboptimal solution, where the efficient solution of the $2 \mathrm{D}$ position of CoW, 3D position of UAV, and the available bandwidth at each one of them can be found. The PSO algorithm generates a set of $(n P)$ random solutions as its starting point, where each solution delivers the BS (i.e., CoW or UAV) position. It seeks to promote candidate solutions iteratively based on each candidate's best experience $\left(P_{\text {best }}(i)\right)$ and candidate's best global experience $\left(G_{\text {best }}\right)$. The particle's locations and velocities are evaluated depending on the best location $\left(P_{\text {best }}(i)\right)$ and the best global location $\left(G_{\text {best }}\right)$, where both are enhanced at each iteration. The velocity term is modified using the approach below:

$$
\begin{aligned}
P_{\text {velocity }}(i)= & w P_{\text {velocity }}(i)+a 1 * \operatorname{rand}(V \text { size }) . \\
& *\left(P_{\text {best }}(i)-P(i)\right)+a 2 * \operatorname{rand}(V \text { size }) . \\
& *\left(G_{\text {best }}(i)-P(i)\right),
\end{aligned}
$$

The factor $w$ is the inertia weight, which is used to adjust the convergence speed, $a_{1}$ and $a_{2}$ are factors that represent the particle's stride size toward its local and global best solutions, and (rand $(V$ size $)$ ) is random positive numbers. Each particle's location is enhanced as follows:

$$
P(i)=P(i)+P_{\text {velocity }}(i)
$$

In the same manner, in ES, the PSO finds the solution in two loops. Initially, the BS that has the priority is illustrated as $B S_{1}$, and it is deployed to serve a set of users (U) from I. The other BS is illustrated as $B S_{2}$, and it is deployed next to 
(i) Input: the lower and upper bounds of decision variable (Varmin, Varmax), construction coefficients $\left(\kappa, \phi_{1}, \phi_{2}\right)$, maximum number of iterations (MIt), population size $(n P)$, total number of users $(I)$, and served users by $\mathrm{BS}_{1}(U)$

(ii) Initialization: $\phi=\phi_{1}+\phi_{2}, X=2 \kappa /\left(\left|2-\phi-\left(\phi^{2}-4 \phi\right)\right|^{0.5}\right), w=X, a_{1}=X \phi_{1}, a_{2}=X \phi_{2}$, $G_{\text {Best.Cost }}=-$ inf ;

(iii) $\mathrm{BS}_{\mathbf{1}}$ Loop:

(iv) for $k \leftarrow 1$ to $|I|$

(v) for $j \leftarrow 1$ to $n P$

(vi) $P(j)=$ unifrnd (Varmin, Varmax, Varsize)

(vii) $P_{\text {Velocity }}(j)=$ zeros (Varsize)

(viii) $P_{\text {Cost }}(j)=$ Objectivefunction $(P(j))$

(ix) $P_{\text {Best }}(j)=P(j)$

(x) $P_{\text {Best.Cost }}(j)=P \operatorname{Cost}(j)$

(xi) if $P_{\text {Best.Cost }}(j)>G_{\text {Best.Cost }}$

(xii) $G_{\text {Best }}=P_{\text {Best }}(j)$

(xiii) end if

(xiv) end

(xv) PSO Loop:

(xvi) for $I t \leftarrow 1$ to MIt

(xvii) for $j \leftarrow 1$ to $n P$

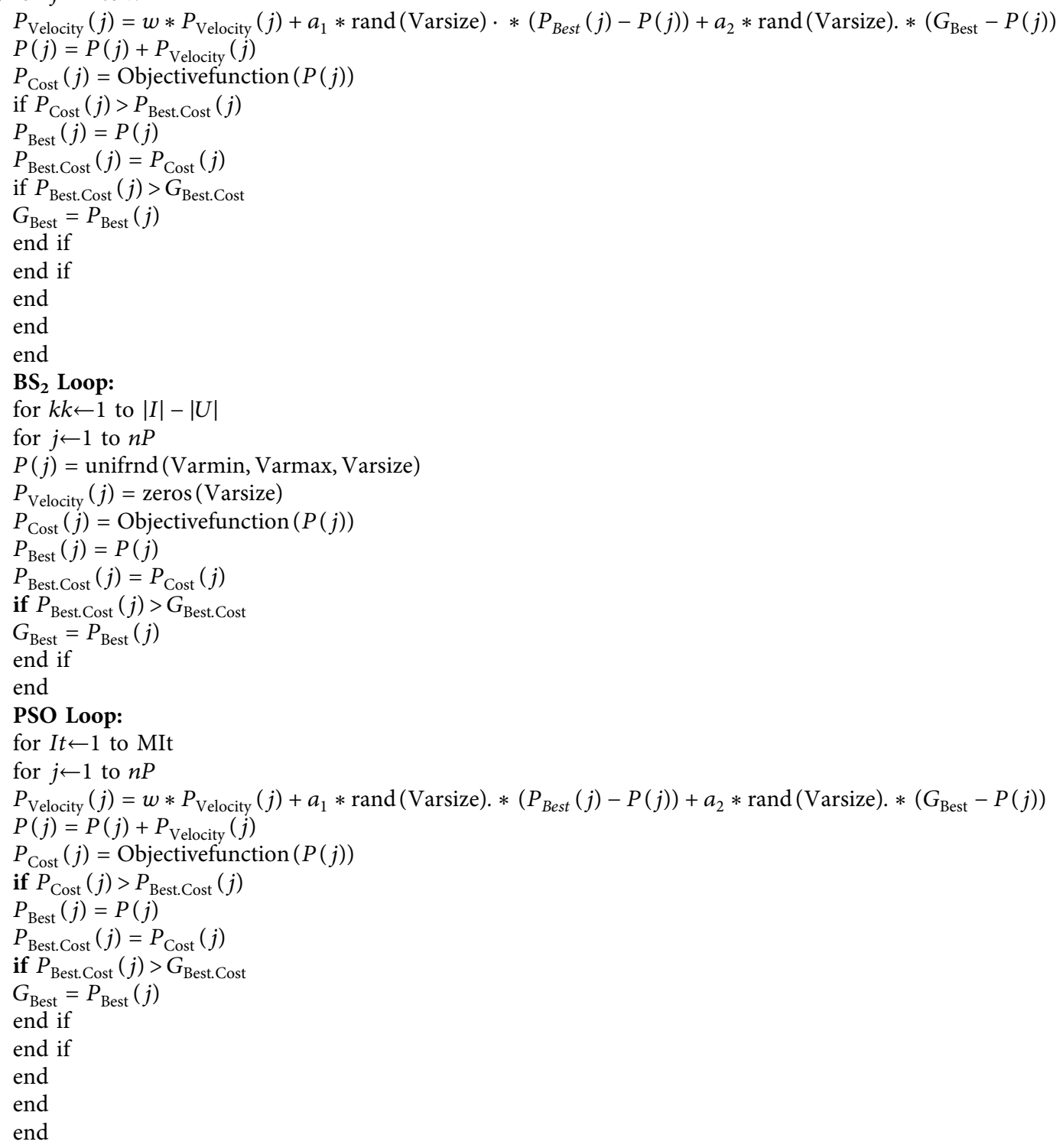


serve as much users as possible from the set $(|I|-|U|)$. The pseudocode for PSO algorithm is described in Algorithm 1:

4.3. Computational Complexity. This section shows the complexity of using either PSO or ES algorithms. It is well known that the ES algorithm finds the optimal solution to the problem. However, its computational complexity is high. Practically, the ES complexity is $O\left(X \times Y \times Z \times\left|U_{u}\right|\right)+O$ $\left(X \times Y \times\left|U_{c}\right|\right)$, where $(X, Y, Z)$ is the $3 \mathrm{D}$ possible locations of the UAV in the proposed area, $\left|U_{u}\right|$ is the number of served users by $\operatorname{UAV},(X, Y)$ is the $2 \mathrm{D}$ possible locations of the CoW in the proposed area, and $\left|U_{c}\right|=|I|-\left|U_{u}\right|$ is the number of unserved users by UAV.

In order to reduce the computational complexity, a suboptimal solution is obtained using heuristic algorithms such as the PSO algorithm. Thus, the PSO complexity is $O\left(n P \times\right.$ MIt $\left.\times\left|U_{u}\right|\right)+O\left(n P \times\right.$ MIt $\left.\times\left|U_{c}\right|\right)$, where $n P$ is a population of random solutions, MIt is the number of iterations, $\left|U_{u}\right|$ is the number of users served by UAV, and $\left|U_{c}\right|=|I|-\left|U_{u}\right|$ is the number of unserved users by UAV.

\section{Performance Evaluations}

Simulation results describing the performance of PSO and exhaustive search algorithms in terms of the served number of users are presented in this section. Simulation results are obtained using MATLAB R2020a. We assume an urban area of $(1000 \mathrm{~m} \times 1000 \mathrm{~m})$, which consists of roads as shown in Figure 1. Furthermore, a total of 200 users are supposed to be uniformly distributed over the given area. We use the evaluation parameters as shown in Table 1 . In addition, the parameters of the PSO algorithm are listed in Table 2.

Figure 2 shows the number of served users versus the percentage of allocated bandwidth for $\mathrm{CoW}(\gamma)$ using the aforementioned algorithms. The figure is obtained when the priority is given to the CoW at the beginning. As shown in the figure, there is a clear trend of decreasing the number of served users of the UAV as its percentage of bandwidth decreases (equivalently, $\gamma$ increases). Similarly, there is an apparent increase in the number of served users of the CoW as its percentage of bandwidth, $\gamma$, increases. As a result, there is an optimal percentage of bandwidth, $\gamma$, where the number of served users is maximized. In this case, the optimal percentage of bandwidth given to the CoW is $\gamma=0.7$, whereas the remaining percentage of bandwidth $(1-\gamma)=0.3$ should be provided to the UAV. In addition, the figure shows that the served number of users of CoW using the PSO algorithm almost coincides with that result once using the exhaustive search algorithm. The reason behind this is the flexibility of CoW to choose its location once it is given priority before the UAV. However, there is a small gap in the served number of users of UAV (as well as the total number of users).

Figure 3 depicts the number of served users in terms of the percentage of bandwidth provided to the CoW, $\gamma$, when the UAV has the priority at the beginning. The figure shows a clear trend of increasing (decreasing) the number of served users of the CoW (UAV) as the percentage of the bandwidth
TABLE 1: Values of the evaluation parameters.

\begin{tabular}{lccccc}
\hline Parameter & $f_{c}$ & $\mathrm{~B}$ & $h_{\mathrm{CoW}}$ & $\left(h_{\min }, h_{\max }\right)$ & $n$ \\
\hline Value & $2 \mathrm{GHz}$ & $40 \mathrm{MHz}$ & $6 \mathrm{~m}$ & $(100 \mathrm{~m}, 300 \mathrm{~m})$ & $(2,4)$ \\
Parameter & $P_{\mathrm{COW}}$ & $P_{u}$ & $P_{i, q}$ & $\mathrm{SNR}_{\text {th }}$ & \\
Value & $40 \mathrm{dBm}$ & $20 \mathrm{dBm}$ & $200 \mathrm{kHz}$ & $20 \mathrm{~dB}$ & \\
\hline
\end{tabular}

TABLE 2: Values of the PSO algorithm's parameters.

\begin{tabular}{lccccc}
\hline Parameter & $\mathrm{nP}$ & MIt & $($ Varmin, Varmax $)$ & Varsize & $\left(w, a_{1}, a_{2}\right)$ \\
\hline Value & 20 & 100 & $(0,1000)$ & 3 & $(1,2.05,2.05)$ \\
\hline
\end{tabular}

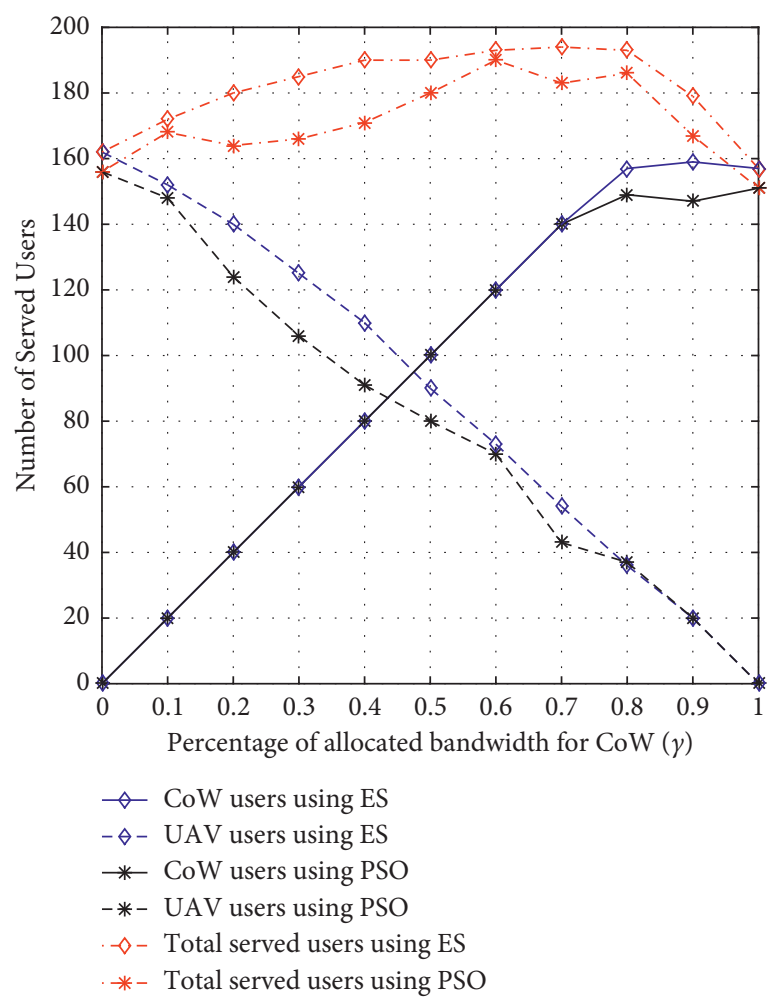

FIgURe 2: Number of served users using PSO and ES algorithms when the priority is given to the CoW at the beginning.

given to the CoW, $\gamma$, increases. As shown in the figure, the served users of the UAV using the PSO algorithm, or exhaustive search algorithm, are the same. That is because the UAV has the priority of choosing its location before the CoW does. In addition, the figure shows that the results of the PSO algorithm can achieve the results of the exhaustive search algorithm with no more than a 5\% deviation. However, the complexity of the PSO algorithm is much lower than the complexity of the exhaustive search algorithm.

To show which BS should be given priority at the beginning, Figure 4 shows the probability of priority provided to the UAV and CoW depending on the percentage of bandwidth provided to the CoW $(\gamma)$. The figure shows that the UAV must prioritize choosing its location before the CoW when its percentage of bandwidth $(1-\gamma)$ is higher than 0.35 (or when $\gamma$ is less than 0.65). In contrast, the CoW must be prioritized at the beginning when its percentage of bandwidth 


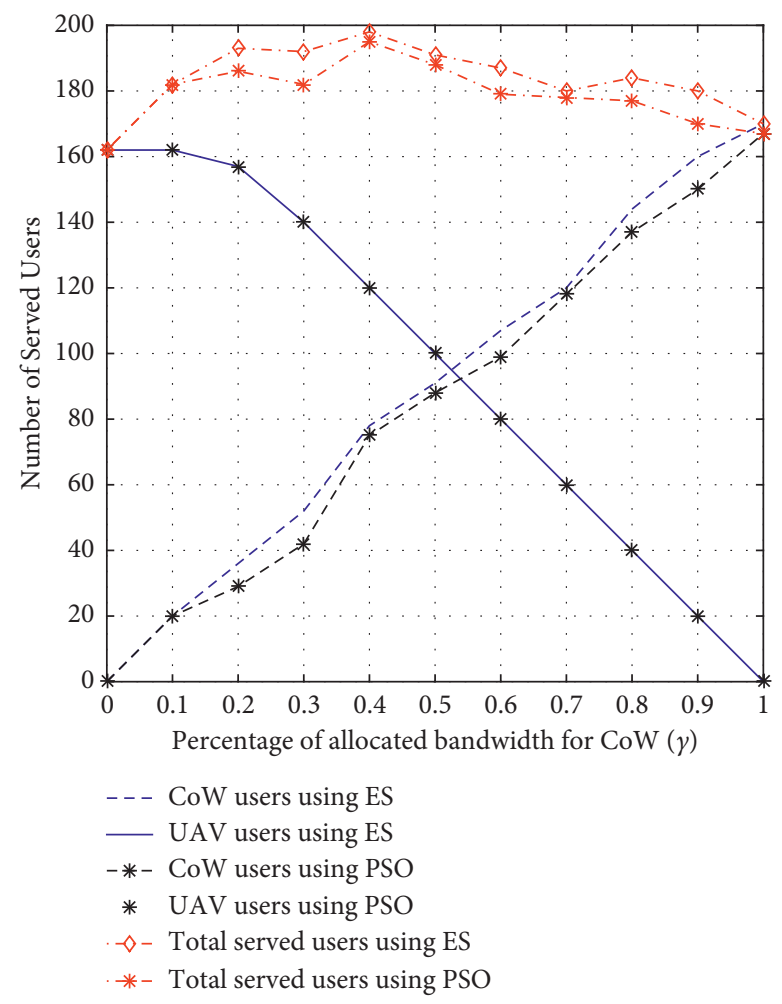

FIgURE 3: Number of served users using PSO and ES algorithms when the priority is given to the UAV at the beginning.

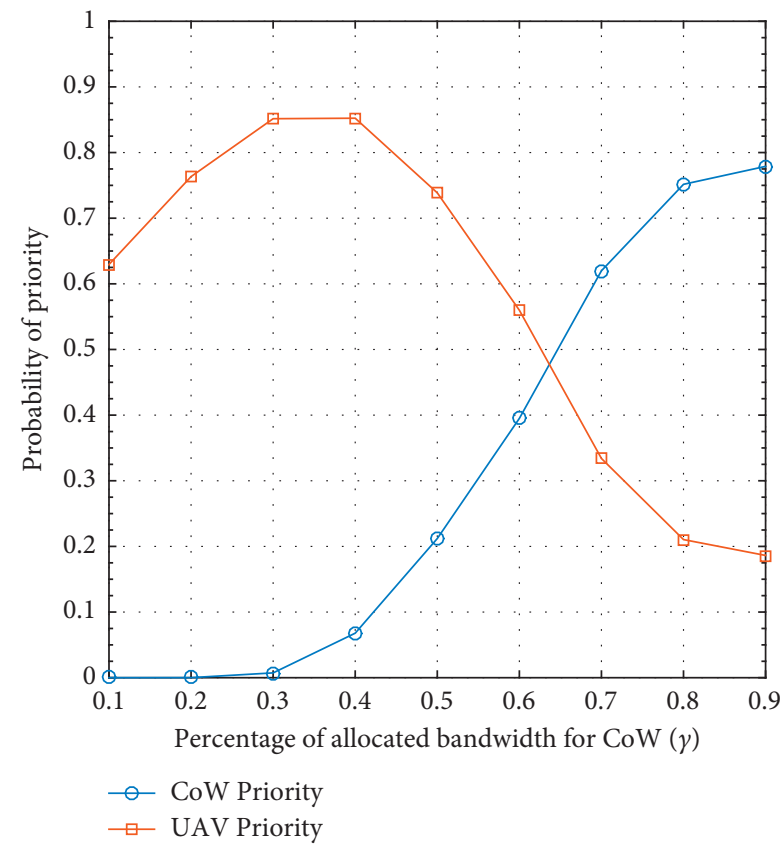

Figure 4: Probability of priority versus the percentage of bandwidth given to the $\mathrm{CoW}(\gamma)$.

$(\gamma)$ is more than 0.65 . Among the plausible explanations for these results is the fact that the UAV has more flexibility of movement than CoW. The CoW can move only on the roads, as shown in Figure 1. However, the UAV can freely move between all of the $3 \mathrm{D}$ possible locations in the given area. It is essential to realize that the priority does not matter in the extreme cases, where CoW is only working (i.e., $\gamma=1$ ) or only $\mathrm{UAV}$ is only working (i.e., $\gamma=0$ ).

Now, to show the optimization results of our proposed algorithm, Figure 5 shows the optimized locations of the UAV (blue square) at $(1000,600,290)$ and CoW (green circle) at $(225,610)$ using the ES algorithm. Note that the height of the $\mathrm{CoW}$ is fixed at $6 \mathrm{~m}$. In this setting, priority is given to the UAV at the beginning with the percentage of bandwidth $(1-\gamma)=0.6$. Thus, the UAV serves 120 users (blue dots), and then CoW serves 78 users (green dots). As a result, $99 \%$ of the users will be served.

Moreover, Figure 6 shows the optimized locations of the CoW (green circle) and UAV (blue square) using the ES algorithm once the priority is given to the $\mathrm{CoW}$ at the beginning. In this case, the best CoW location is $(610,343)$ with the percentage of the bandwidth of $\gamma=0.7$ and then the best UAV location of the UAV is $(400,750,290)$ with the percentage of bandwidth $(1-\gamma)=0.3$. Accordingly, the CoW serves 140 users, and then the UAV serves 54 users. Therefore, $97 \%$ of users are served under this setting.

To show the computational complexity of both ES and PSO algorithms, Table 3 shows the running time of each algorithm under different values of the bandwidth percentage provided to the CoW $(\gamma)$. As it can be seen from the table, the PSO algorithm improves the required time significantly. In addition, note that these results of the ES algorithm are obtained at $50 \mathrm{~m}$ step size. If the step size is decreased to locate the UAV and CoW precisely, the running time will be extremely increased. For example, if the step size is $25 \mathrm{~m}$ and $\gamma=0.6$, the running time will be 963.5 seconds. 


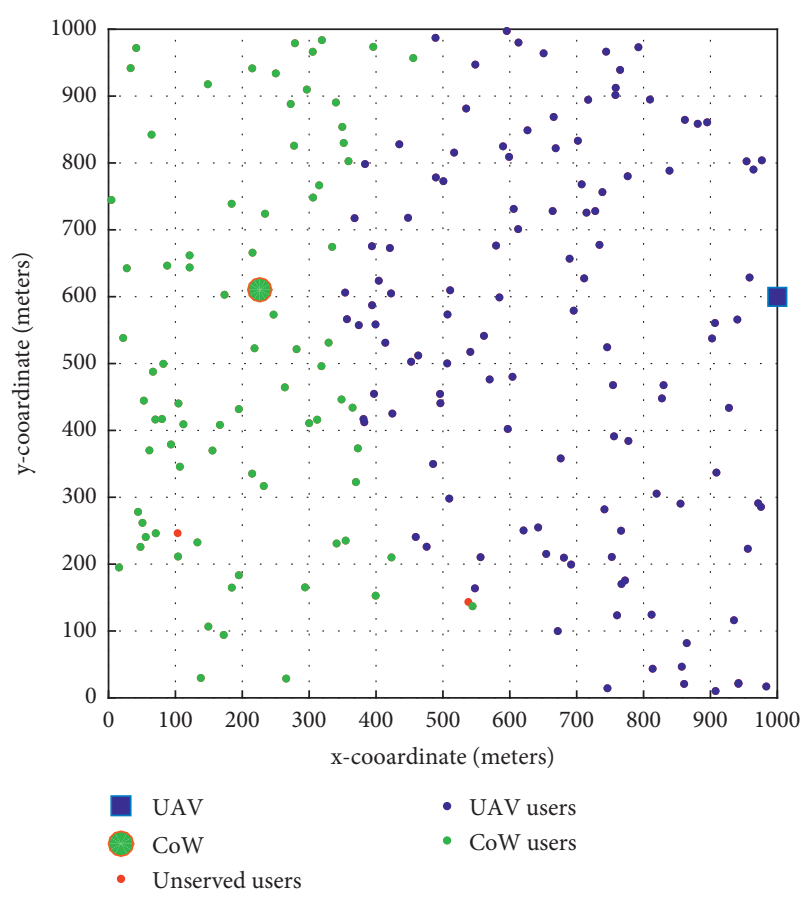

FIgUre 5: Deployment of the CoW and UAV using ES algorithm when the priority is given to the UAV at the beginning.

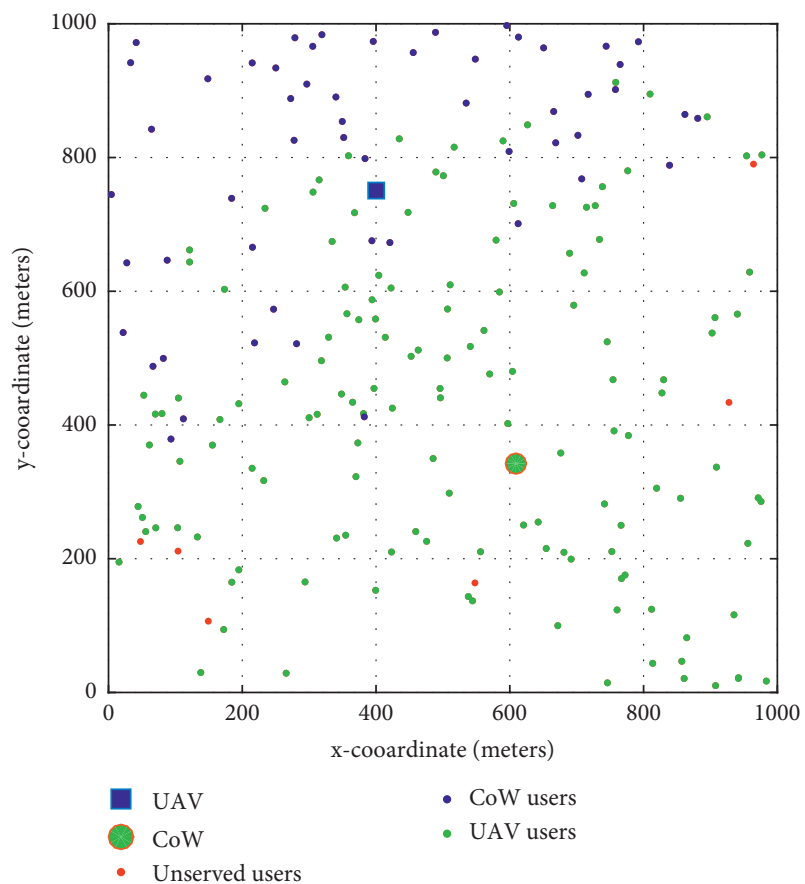

FIgURE 6: Deployment of the CoW and UAV using ES algorithm when the priority is given to the CoW at the beginning.

Finally, to show the robustness of our proposed algorithm, we study its performance over three different users' distributions. The first distribution $\left(D_{1}\right)$ is, what we have used, overall evaluations. The second distribution $\left(D_{2}\right)$ is a random distribution. The third distribution $\left(D_{3}\right)$ is a uniform distribution, where the users are distributed uniformly
TABLE 3: Running time (in seconds) of the PSO and ES algorithms.

\begin{tabular}{lcccc}
\hline$\gamma$ & 0 & 0.4 & 0.6 & 1 \\
\hline PSO & 20.37 & 20.56 & 20.78 & 21.01 \\
ES & 128.16 & 128.91 & 131.16 & 153.78 \\
\hline
\end{tabular}

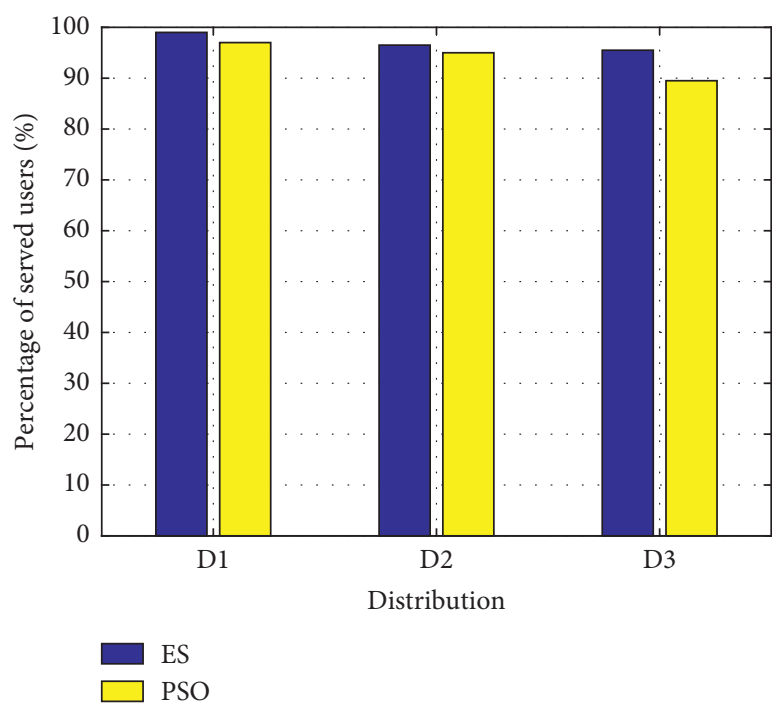

FIGURE 7: Robustness of the proposed algorithm.

over the given area. Figure 7 shows the performance over all the three aforementioned distributions. It can be seen that a very low change in the served users percentage would have occurred. In addition, in the impractical scenario where the distribution is uniform (i.e., $\mathrm{D}_{3}$ ), the dropping in the served users percentage would be at minimum, around $5 \%$.

\section{Conclusion}

This paper proposes a cooperative scheme between a CoW and a UAV intending to maximize the wireless coverage in emergencies. First, we found which one of the CoW and UAV must be located at the beginning. Then, the best $2 \mathrm{D}$ location for the CoW and the 3D location for the UAV are optimized. In addition, the percentage of the available bandwidth that must be provided for each base station (CoW and UAV) is calculated. Finally, we found that the priority depends on the percentage of the bandwidth given to each base station. For CoW-UAV system, the simulation results show that the UAV must prioritize choosing its location before the CoW when its percentage of bandwidth $(\gamma)$ is less than 0.65 . In contrast, the CoW must be prioritized at the beginning when its percentage of bandwidth $(\gamma)$ is more than 0.65 . In addition, the simulation results show that the PSO algorithm can achieve the results of the exhaustive search algorithm with no more than a $5 \%$ deviation. However, the complexity of the PSO algorithm is much lower than the complexity of the exhaustive search algorithm.

\section{Data Availability}

Data are available from the corresponding author upon request. 


\section{Conflicts of Interest}

The authors declare that they have no conflicts of interest.

\section{Acknowledgments}

This research work was supported by the Office of Research and Innovation at Alfaisal University, Saudi Arabia.

\section{References}

[1] X. Zhang and L. Duan, "Fast deployment of uav networks for optimal wireless coverage," IEEE Transactions on Mobile Computing, vol. 18, no. 3, pp. 588-601, 2018.

[2] H. Shakhatreh, A. Alenezi, A. Sawalmeh, M. Almutiry, and W. Malkawi, "Efficient placement of an aerial relay drone for throughput maximization," Wireless Communications and Mobile Computing, vol. 2021, 2021.

[3] M. Mozaffari, W. Saad, M. Bennis, Y.-H. Nam, and M. Debbah, "A tutorial on uavs for wireless networks: Applications, challenges, and open problems," IEEE Communications Surveys \& Tutorials, vol. 21, no. 3, pp. 2334-2360, 2019.

[4] A. Sawalmeh, N. Othman, and H. Shakhatreh, "Efficient deployment of multi-uavs in massively crowded events," Sensors, vol. 18, no. 11, Article ID 3640, 2018.

[5] N. Namvar, A. Homaifar, A. Karimoddini, and B. Maham, "Heterogeneous uav cells: An effective resource allocation scheme for maximum coverage performance," IEEE Access, vol. 7, pp. 164708-164719, 2019.

[6] A. H. Sawalmeh, N. S. Othman, H. Shakhatreh, and A. Khreishah, "Wireless coverage for mobile users in dynamic environments using uav," IEEE Access, vol. 7, pp. 126376-126390, 2019.

[7] W. D. Ivancic, R. J. Kerczewski, R. W. Murawski, K. Matheou, and A. N. Downey, "Flying drones beyond visual line of sight using 4g lte: Issues and concerns," in Proceedings of the 2019 Integrated Communications, Navigation and Surveillance Conference (ICNS), pp. 1-13, Herndon, VA, USA, April 2019.

[8] X. Zhang and L. Duan, "Optimization of emergency uav deployment for providing wireless coverage," in Proceedings of the GLOBECOM 2017-2017 IEEE Global Communications Conference, pp. 1-6, Singapore, December 2017.

[9] N. Zhao, W. Lu, M. Sheng et al., "Uav-assisted emergency networks in disasters," IEEE Wireless Communications, vol. 26, no. 1, pp. 45-51, 2019.

[10] M. Deruyck, J. Wyckmans, W. Joseph, and L. Martens, "Designing uav-aided emergency networks for large-scale disaster scenarios," EURASIP Journal on Wireless Communications and Networking, vol. 2018, no. 1, pp. 1-12, 2018.

[11] G. Liu, H. Shakhatreh, A. Khreishah, X. Guo, and N. Ansari, "Efficient deployment of uavs for maximum wireless coverage using genetic algorithm," in Proceedings of the 2018 IEEE 39th Sarnoff Symposium, pp. 1-6, Newark, NJ, USA, September 2018.

[12] L. Rabieekenari, K. Sayrafian, and J. S. Baras, “Autonomous relocation strategies for cells on wheels in environments with prohibited areas," in Proceedings of the 2017 IEEE International Conference on Communications (ICC), pp. 1-6, Paris, France, May 2017.

[13] D. Abusch-Magder, P. Bosch, T. E. Klein, P. A. Polakos, L. G. Samuel, and H. Viswanathan, "911-now: A network on wheels for emergency response and disaster recovery operations," Bell Labs Technical Journal, vol. 11, no. 4, pp. 113-133, 2007.

[14] J.-S. Huang and Y.-N. Lien, "Challenges of emergency communication network for disaster response," in Proceedings of the 2012 IEEE International Conference on Communication Systems (ICCS), pp. 528-532, Singapore, November 2012.

[15] M. Gapeyenko, V. Petrov, D. Moltchanov, S. Andreev, N. Himayat, and Y. Koucheryavy, "Flexible and reliable uavassisted backhaul operation in $5 \mathrm{~g}$ mmwave cellular networks," IEEE Journal on Selected Areas in Communications, vol. 36, no. 11, pp. 2486-2496, 2018.

[16] K. F. Hayajneh, K. Bani-Hani, H. Shakhatreh, M. Anan, and A. Sawalmeh, "3d deployment of unmanned aerial vehiclebase station assisting ground-base station," Wireless Communications and Mobile Computing, vol. 2021, p. 2021.

[17] D. Sikeridis, E. E. Tsiropoulou, M. Devetsikiotis, and S. Papavassiliou, "Wireless powered public safety iot: A uavassisted adaptive-learning approach towards energy efficiency," Journal of Network and Computer Applications, vol. 123, pp. 69-79, 2018.

[18] P. Vamvakas, E. E. Tsiropoulou, and S. Papavassiliou, "Riskaware resource management in public safety networks," Sensors, vol. 19, no. 18, Article ID 3853, 2019.

[19] N. Patrizi, G. Fragkos, E. E. Tsiropoulou, and S. Papavassiliou, "Contract-theoretic resource control in wireless powered communication public safety systems," in Proceedings of the GLOBECOM 2020-2020 IEEE Global Communications Conference, pp. 1-6, Taipei, Taiwan, December 2020.

[20] T. S. Rappaport, Wireless communications: principles and practice, Vol. 2, Prentice hall PTR, , New Jersey, NY, USA, 1996.

[21] A. Goldsmith, Wireless communications, Cambridge university press, Cambridge, England, 2005.

[22] A. Al-Hourani, S. Kandeepan, and S. Lardner, "Optimal lap altitude for maximum coverage," IEEE Wireless Communications Letters, vol. 3, no. 6, pp. 569-572, 2014.

[23] M. Mozaffari, W. Saad, M. Bennis, and M. Debbah, "Drone small cells in the clouds: Design, deployment and performance analysis," in Proceedings of the 2015 IEEE global communications conference (GLOBECOM), pp. 1-6, San Diego, CA, USA, December 2015.

[24] R. Eberhart and J. Kennedy, "A new optimizer using particle swarm theory," in Proceedings of the MHS'95. Proceedings of the Sixth International Symposium on Micro Machine and Human Science, pp. 39-43, Nagoya, Japan, October 1995.

[25] Y. Shi and R. Eberhart, "A modified particle swarm optimizer," in Proceedings of the 1998 IEEE international conference on evolutionary computation proceedings. IEEE world congress on computational intelligence (Cat. No. 98TH8360), pp. 69-73, Anchorage, AK, USA, May 1998. 